\title{
Efficacy of Trans-abdominis Plane Block for Post Cesarean Delivery Analgesia in Low-income Countries: a Phase Three Feasibility Study.
}

Evans Azina Sanga ( $\sim$ evansnga@hotmail.com )

Kilimanjaro Christian Medical Center https://orcid.org/0000-0002-6191-6974

Ansbert Sweetbert Ndebea

Kilimanjaro Christian Medical Centre

Shuweikha Salim

Kilimanjaro Christian Medical Centre

Mwemezi Kaino

CURE international Hospital

\section{Bernard Njau}

Kilimanjaro Christian Medical University College

\section{Rogers Temu}

Kilimanjaro Christian Medical Centre

Research article

Keywords: Trans Abdominis Plane block, Pain and Caesarean section

Posted Date: December 7th, 2020

DOI: https://doi.org/10.21203/rs.3.rs-120194/v1

License: (c) (1) This work is licensed under a Creative Commons Attribution 4.0 International License. Read Full License 


\section{Abstract}

Background: Optimal pain control in a parturient woman undergoing caesarean section is essential for preventing complications such as venous thrombo-embolism and improving maternal satisfaction, early functional recovery, mother-baby bond and breastfeeding. Intentional pain assessment and adequate management to acceptable pain severity using multimodal methods can be achieved in low-middle income countries (LMICs).

Aim: Is to assess the efficacy of transversus abdominis plane (TAP) block and satisfaction post cesarean delivery analgesia at Kilimanjaro Christian Medical Centre in Low-Income countries.

Methods: The study population consisted of 72 participants who met criteria posted for elective and emergency caesarean section. They were blindly assigned into two groups: group A was the interventional group which received TAP block and standard pain management according to local protocols and consisted of 41 participants and group $B$ was the control group which received standard pain management without TAP block and consisted of 31 participants. In Group A 30ml of $0.25 \%$ bupivacaine single shot was deposited in the TAP plane bilaterally for postoperative analgesia. Participants were randomized using a parallel method. Their demographics were recorded before surgery and visual analogue scale was used to assess postoperative pain at rest and on movement, and maternal satisfaction at $0 \mathrm{hrs}, 6 \mathrm{hrs}, 12 \mathrm{hrs}$ and $24 \mathrm{hrs}$.

Results: Total of 72 patients were analyzed using NRS with pain score at $0 \mathrm{hr}, 6 \mathrm{hr}$ and $12 \mathrm{hr}$ was significantly low by about $50 \%$ in Intervened group as compared to control group with (p-value ( 2 tail) of < 0.001 however at $24 \mathrm{hrs}$. was 0.272 . Participant in group A had extra movements at $0 \mathrm{hr}, 6 \mathrm{hrs}$ and $12 \mathrm{hrs}$ with $p$-value $<0.001$ as compare to control cut had no significant difference when coughing. Martenal satisfaction with pain management was $95.1 \%$ with no reported adverse event.

Conclusions: Trans Abdominis Plane block when used as part of multimodal pain management is more effective in managing post cesarean pain resulting in less physical limitation and high martenal satisfaction.

Trail registration: Register in Pan African Clinical Trial Registry (PACTR) with no PACTR202011815473426 on 12 November 2020.

\section{Background}

The rate of caesarean section has been increasing worldwide and approximately 18.5 million are done annually. On average, at Kilimanjaro Christian Medical Center (KCMC), a referral teaching hospital in northern Tanzania13 deliveries are done each day, of which $49.2 \%$ are by $\mathrm{C} / \mathrm{S}$. With a gift of a newborn a parturient needs to be alert, comfortable and have early functional recovery. However, Pain is one of the immediate post-operative outcomes with prevalence of more than 78.4\% (moderate to severe) [1][2][3]. 
When pain is not optimally treated the mother will be at an increased risk of delay ambulation, thromboembolic event, delayed wound healing, hemorrhages, stress, hemodynamic derangement, myocardial ischemia and shallow breathing which could result into hypoxia, hypercarbia and respiratory infections. As a result of these factors the newborn could have reduced mother-child bond and difficulty breastfeeding [4][5][6].

There is no study on post cesarean pain that was done in Tanzania but few studies on general postoperative pain report that pain was under treated and more than $80 \%$ of post-operative patients reported moderate to severe pain. In developed countries, epidural is the mainstay technique for labour analgesia but it is labour intense, requires strict monitoring and is associated with more complication. Other techniques include bolus doses for paravertebral, transversus abdominis plane block (TAP) and erector spinae block with similar efficacy but less risk for complications. TAP block was first described in 1993 and it involves deposition of local anaesthetic between the posterior aponeurosis of the internal oblique muscle and the aponeuroses of the transversus abdominis muscle [7][4].

Local protocol at KCMC indicates administration of paracetamol, NSAID and opioids alone for management of post cesarean delivery pain. Regardless of the data paucity in LMICs on regional techniques for post caesarian pain management, it is appreciated that post cesarean delivery pain is under treated. Parallel to the efforts to improve pain management at KCMC the aim of this study was to determine the feasibility of trans abdominis plane block for post cesarean delivery analgesia in the resource-constrained environment.

\section{Methodology}

\section{Study design}

A hospital based parallel design, phase three two armed feasibility study conducted from 1st May to 31 st June 2019 at KCMC referral hospital obstetrics operating room 1 and 2 and in the post ceasarean wards. $\mathrm{KCMC}$ is a 700-bed tertiary teaching hospital with a catchment population of 15 million and is located in northern Tanzania. KCMC has 55 obstetric beds and on average 4 cesarean sections per day are performed (KCMC report, 2018).

Ethical approval was obtained from the Research Ethics Review Committee of the Kilimanjaro Christian Medical University College (KCMUCo) with certificate no 2394 and permission to conduct the study was granted by the KCMC hospital. Trail was Register in Pan African Clinical Trial Registry (PACTR) with no PACTR202011815473426 on 12 November 2020.

\section{Participants}

\section{Inclusion criteria}


All parturient participants classified as ASA II and III planned for cesarean section at KCMC during the study period and concented were enrolled in this study.

\section{Exclusion criteria}

Participants with communication difficulties, obesity ( $\mathrm{BMI}>30$ ), any contraindication of spinal anaesthesia, allergic to amino-amide local anesthetics such as bupivacaine, coagulopathy, and local skin infection at any injection sites.

\section{Interventions}

All parturient women planned for emergency or elective caesarian section who met the inclusion criteria were recruited to join the study. Initially, the demographic data was extracted from the parturient files into the data sheets and then they were presented with all the information regarding the study. This information included benefits and disadvantages of the study and all their questions were satisfactorily responded to and finally, they were asked to sign the consent. They were informed refusal to consent would not jeopardize further treatment at KCMC.

During preparation for cesarean section all participants were treated as per KCMC Obstetrics protocols with an exception that ultrasound-guided TAP block was administered to participants in group A. In the operating room, patients were placed on left lateral position and monitors were placed to obtain baseline vitals of the parturient and the fetus. Then IV pre-loading with $500 \mathrm{ml}$ to $1000 \mathrm{ml}$ Ringers solution was started and pre-operative antibiotic was administered. They were all operated under spinal anaesthesia using a G25 LUCKmed quinkle needle with $2 \mathrm{ml}(10 \mathrm{mg})$ of $0.5 \%$ heavy bupivacaine (BUPICAN $0.5 \%$ Claris $4 \mathrm{ml}$ ampule). All spinal anaesthesia were administered in seating position. Before surgery participants were randomized into groups $A$ and $B$ by picking an envelope from a box. After surgery Group A was subjected into intervention as per study protocol..

\section{Procedure}

At the end of the surgery, bilateral ultrasound guided TAP block was performed by the principal investigator using $60 \mathrm{ml}$ of $0.25 \%$ plain bupivacaine (BUPICAN 0.5\% Claris $20 \mathrm{ml}$ vial), $30 \mathrm{ml}$ each side. The bupivacaine aliquots were prepared by mixing $15 \mathrm{ml}$ of $0.5 \%$ bupivacaine with $15 \mathrm{ml}$ of water for injection at the bed side and the block was administered using aseptic non-touch technique (ANTT).

After preparing the skin with an antiseptic solution, a linear high frequency ultrasound probe $(6-13 \mathrm{MHz}$, Sonosite $\mathrm{M}$ - Turbo®) was placed transversely on the anterolateral abdominal wall between the iliac crest and the costal margin. The three layers of muscles i.e. external oblique, internal oblique, and the transversus abdominis were identified. To assist with identifying these structures, the probe was moved anteriorly to the rectus sheath and the fascial planes were followed laterally .

A sterile 22-gauge, 115-mm Quincke needle was attached to a syringe containing $10 \mathrm{ml}$ water for injection via a flexible tube connector. The sterile water was used to locate the position of the tip of the needle in 
the fascial plane by hydro dissection. The needle was introduced through the skin anteriorly in the plane of the ultrasound beam and advanced further into the fascial plane between the internal oblique and transversus abdominis muscles with its tip lying in the mid axillary line. After aspiration to exclude inadvertent vascular puncture, $2-5 \mathrm{ml}$ of sterile water was used to separate the fascial planes. Then $30 \mathrm{ml}$ of the aliquot was administered while closely observing for signs (tinnitus, perioral numbness, metallic taste in the mouth) and symptom (slurring of speech, mental status changes, increase in QT,widen QRS, ectopic beats and hypotension) of toxicity. TAP block was performed in a similar fashion on the opposite side.

After completion of the procedure, patients were shifted to the recovery for 30 minutes monitoring before they were transferred to the obstetric ward. Both groups received post-operative pain treatment according to the KCMC protocol.

Pain was assessed by a blinded independent observer immediately after delivery (0hr), 6hrs, $12 \mathrm{hrs}$ and 24 hrs.

\section{Outcomes}

\section{Primary outcomes}

Primary outcomes were the pain score, which was assessed by using numerical rating scale (NRS) and function assessment by using pain scores on physical activities such as turning in bed, sitting, standing, walking and coughing. Patients were asked to rate the intensity of pain out of ten using NRS. It a 10-point numerical rating scale with end points representing the extremes of the pain experience: $0=$ "no pain at all" and $10=$ "worst possible pain". The score was classified as mild pain when the score is 0 to 3 , moderate pain when the score is 4 to 7 and severe pain when the score is 8 to 10 [7]

NRS is the most commonly recommended tool in pain studies due to better responsiveness and compliance, easy to use and applicababilty in most settings compared with visual analog scale/verbal rating scale (VAS/VRS) [8]. It is difficult to assess pain objectively, although it can be assessed at rest and during movement by NRS to detect changes and difference in pain scores. Functional assessment was assessed by functional activity including turning in bed, sitting, standing and walking was marked on a numeric rating scale ranging from zero $=$ "no pain" to $10=$ "maximum pain". [9]. Thus, all these two were assessed at $0 \mathrm{hrs}, 6 \mathrm{hrs}, 12 \mathrm{hrs}$ and $24 \mathrm{hrs}$.

\section{Secondary outcome}

Patients satisfaction, question was adopted from the 5-point Likert scale ranging from $1=$ very dissatisfied to $5=$ Very satisfied. Participant were asked two questions on were they satisfied with information of TAP block and 24 pain management. It improves learners' perceptions of readiness, knowledge and prioritization skills by 30-45\% (McGillion et al., 2011; Farooq, Khan and Ahmed, 2016). A pilot study was conducted with 20 patients to validate the data collecting tool and further categorized 
into 3-point Likert scale for easy analysis. And complications of TAP block were assessed such as LAST which was light headedness, blurred vision, tinnitus, metallic test on the tongue, confusion and loss of consciousness. Patients satisfaction was assessed at $24 \mathrm{hrs}$ and complications were assessed all the time up to $24 \mathrm{hrs}$.

\section{Criterial for stop intervention}

Criteria for stop intervention were refusal by the participant at any point in time, sign of LAST which was Lighted headedness, blurred vision, Tinnitus, metallic test on the tongue, confusion and loss of consciousness and those transferred to intensive care unit (ICU). No other medications or procedures were discontinued, denied, delayed or prevented as a result of an intervention of this study.

\section{Sampling}

For study with $80 \%$ power to detect $30 \%$ difference VAS pain score between two groups P-value 0.05 for clinically significant. And standard deviation of 10 with $10 \%$ fall out a sample size of 68 participants (34 to each groups) [12].

\section{Randomization}

Randomization was done by using numbers (from 1 to 86 ) that were written on a piece of paper which was enclosed in 86 envelopes. These envelopes were mixed and put in one pool from which each participant picked one envelop to disclose the number to the principal investigator. Patients who picked even numbers were assigned to group $A$ (intervention group) and those with odd numbers were assigned to group $B$ (control group). with parallel design through number from 1 to 80 all even numbers were group A (TAP group) and odd numbers were assigned group be (control group).

\section{Blinding}

Blinding was done complete research assistant who was going to assess the participant. Although full blinding of participants to condition in this study was not possible, several strategies were employed to reduce the risk of bias. First, information of the study hypothesis was provided to participant. Second participants were clearly instructed only to contact the investigator personally using a phone number provided to them by the investigator and direct to him alone all queries regarding the intervension, to avoid contact with the research assistant for any scheduling concerns, questions regarding intervention.

\section{Data Analysis}

During data collection, special codes were used in place of parturient name then it was double entered into data collecting sheet. Later on, it was cleaned and analyzed using SPSS V24. Descriptive statistics was carried out to describe the socio-demographic and clinical characteristics of the participants were summarized in form proportions, frequency tables and bar charts for categorical variables and Mean and standard deviation used to for continuous. Bivariate analysis using Chi-square test was used to find out the association followed by a logistic regression. Independent t-test was used to compare mean pain 
score between different groups of patients. Statistical differences was determined at $95 \%$ confidence interval with a $\mathrm{P}<0.05$.

\section{Results}

A total of 72 participants met the criteria were recruited. 12 participants were removed as 3 did not wish to continue. 3 were sent to ICU after surgery of obesity, 4 with hemodynamic instability and 2 participants didn't visualize layer to identify transversus abdominis plane to deposit local anesthetics. Thus 41 were in TAP group and 31 in non-intervened.

From Table 1 presents the demographic characteristics of participants, seventy eight percent $(n=32 / 41)$ of participants aged $20-34$ years old were in the intervention group compared with $83.9 \%(n=26 / 31)$ in control groups. The mean \pm standard deviation of the age was $(29 \pm 5.4)$. Chagga were $43.9 \%(n=18 / 41)$ in TAP group and $71 \%(n=22 / 31)$ in the control group, $36.6 \%(n=15)$ were had reached to college level in TAP group while $38.7 \%(n=12)$ had secondary education in Control group.92.7\% $(n=38)$ participants were ASA II in the TAP group and $90.3 \%(n=28)$ in control group, $44 \%(n=28)$ participants were overweight among TAP group and 51.6\%(n=16) from the control group. 53.7\%(n=22) in TAP group came in Labor while $51.6 \%(n=16)$ were in the control group. Tribe only was statistically significant between TAP group and control with P-value of 0.05 .

Table 1.: Demographic characteristics of participants

\begin{tabular}{lccc}
\hline Characteristics & $\begin{array}{c}\text { TAP Group } \\
\mathrm{n}=41(\%)\end{array}$ & $\begin{array}{c}\text { Control Group } \\
\mathrm{n}=31(\%)\end{array}$ & P-value \\
\hline Age & & & \\
<20 yrs. old & $2(4.9)$ & $2(4.9)$ & 0.25 \\
20-34 yrs. old & $32(78)$ & $26(83.9)$ & \\
$\quad>35$ yrs. old & $7(17.1)$ & $3(9.7)$ & \\
Tribe & & & \\
Masai & $4(9.8)$ & $1(3.2)$ & 0.05 \\
Chagga & $18(43.9)$ & $22(71)$ & \\
Sambaa & $3(7.3)$ & $1(3.2)$ & \\
Others & $16(39)$ & $7(22.6)$ & \\
Education Level & & & \\
No Education & $1(2.4)$ & $0(0)$ & \\
Primary & $12(29.3)$ & $8(25.8)$ & \\
Secondary & $13(31.7)$ & $12(38.7)$ & 0.78 \\
College & $15(36.6)$ & $11(35.5)$ & \\
ASA Classification & & & \\
ASA II & $38(92.7)$ & $28(90.3)$ & \\
ASA III & $3(7.3)$ & $3(9.7)$ & 0.72 \\
BMI & $12(30)$ & $15(48.4)$ & \\
Normal Weight & $28(44)$ & $16(51.6)$ & 0.65 \\
Over weight & & & \\
Labour pain & $22(53.7)$ & $16(51.6)$ & \multirow{2}{*}{0.86} \\
Yes & $19(46.4)$ & $15(48.4)$ & \\
No & & &
\end{tabular}




\section{Pain score at rest}

Table 2 presents the NRS of pain at rest, the scores were different in post anaesthesia care unity (PACU) at 0 hour both groups but were significantly lower at all- time up to $24 \mathrm{~h}$ in TAP group compared to control group. By using ANOVA, pain score at $0 \mathrm{hr}, 6 \mathrm{hr}$ and $12 \mathrm{hr}$ was significantly low by about $50 \%$ in TAP group with compared with the control group with ( $p$-value $(2$ tail) of $<0.001)$. However, at 24 hrs. there was a small difference between the two groups with ( $p$-value $(2$ tail $)=0.272)$.

Table 2: Pain score at $0 \mathrm{hr}, 6 \mathrm{hrs}, 12 \mathrm{hrs}$ and 24 at rest

\begin{tabular}{cccc}
\hline Time & $\begin{array}{c}\text { TAP } \\
(\text { Mean } \pm \text { SD })\end{array}$ & $\begin{array}{c}\text { Control } \\
(\text { Mean } \pm \text { SD })\end{array}$ & p-value \\
\hline Pain score at: & & & \\
0 & $1.6 \pm 2.18$ & $4.5 \pm 2.18$ & $<0.001$ \\
6 & $2.8 \pm 1.38$ & $4.9 \pm 1.74$ & $<0.001$ \\
12 & $2.5 \pm 1.42$ & $4.3 \pm 1.43$ & $<0.001$ \\
24 & $2.4 \pm 1.22$ & $2.7 \pm 1.26$ & 0.272 \\
\hline
\end{tabular}

\section{Pain score on physical activities}

Table 3 showing pain score on physical activities using NRS of pain. Since about $94.4 \%$ were given spinal anaesthesia and we didn't interfere post op KCMC hospital protocol, which says the patient will not walk until $12 \mathrm{hrs}$, hence was not accessed at $0 \mathrm{hr}$ and $6 \mathrm{hrs}$. Participants in TAP group had more movements $0 \mathrm{hr}$, $6 \mathrm{hrs}$ and $12 \mathrm{hrs}$ with $\mathrm{p}$-value $<0.001$ as compared to control, which was reduced ( such as could not turn on bed or sit). However, the was no significant difference between the groups at 24hrs ( $\mathrm{p}$-value $=0.283$ ).

Table 3: Pain score at $0 \mathrm{hr}, 6 \mathrm{hrs}, 12 \mathrm{hrs}$ and 24 on physical movement 


\begin{tabular}{cccc}
\hline Characteristics & $\begin{array}{c}\text { TAP } \\
\text { Mean } \pm \text { SD })\end{array}$ & $\begin{array}{c}\text { Control } \\
\text { Mean } \pm \text { SD })\end{array}$ & p-value \\
\hline Pain score on movement & & & \\
0 & $0.6 \pm 1.83$ & $2.1 \pm 2.88$ & 0.009 \\
6 & $2.5 \pm 1.89$ & $4.4 \pm 2.46$ & 0.018 \\
12 & $2.3 \pm 1.72$ & $3.9 \pm 1.99$ & 0.002 \\
24 & $2.2 \pm 1.22$ & $2.3 \pm 1.50$ & 0.680 \\
NRS on sleeping & $0.7 \pm 1.88$ & $2.0 \pm 2.66$ & 0.002 \\
0 & $2.0 \pm 1.62$ & $3.4 \pm 1.99$ & $<0.001$ \\
12 & $1.6 \pm 1.56$ & $2.5 \pm 1.61$ & $<0.001$ \\
24 & $1.4 \pm 1.31$ & $1.4 \pm 1.50$ & 0.950 \\
0 & $1.3 \pm 2.21$ & $3.3 \pm 2.83$ & 0.001 \\
6 & $2.5 \pm 1.31$ & $4.3 \pm 2.25$ & 0.024 \\
12 & $1.8 \pm 1.41$ & $3.0 \pm 1.88$ & 0.004 \\
24 & $1.4 \pm 1.18$ & $1.7 \pm 1.74$ & 0.023 \\
NRS on deep breath & & & \\
6 & $1.4 \pm 2.19$ & $3.7 \pm 2.67$, & 0.001 \\
12 & $2.7 \pm 1.56$ & $4.5 \pm 2.43$ & 0.020 \\
24 & $2.7 \pm 1.91$ & $3.4 \pm 1.89$ & 0.023 \\
NRS on coughing & $1.9 \pm 1.41$ & $2.3 \pm 1.70$ & 0.237
\end{tabular}

\section{Patients' satisfaction}

Patients' satisfaction was categorized into 3-point Likert scale satisfied, neither and dissatisfied. All participants were given information about TAP block and pain management from which $97.6 \%$ and $96.8 \%$ were satisfied about the information given to them in TAP and control group ( $p$-value $=0.81)$. There was no statistical difference between them as graph 2. However, after block $95.1 \%$ of a participant in the TAP group were satisfied with their pain management as compared to $54.8 \%$ in the control group as in graph 3 treated by systemic oral analgesia. Of these complications of TAP block; LAST, nausea vomiting and dizziness explored in the study There was no complication related to TAP block was reported in this study.

\section{Discussion}

Caesarean section is increasing significantly more recently, pain post-operative being among immediate consequence. Pain management after CS is aiming at conferring maternal comfort, no side effect to mother and a born child and early recuperate to normal function to help a new born baby. However, there is a challenge in achieving this due to fact that is associated with lots of poor pain control, nausea and vomiting [13]. Local regional technique such as TAP have been studied and compared to other techniques to incorporate as component in multimodal pain management approach proved efficacious [14].

\section{Pain score at rest.}

The results in this study have demonstrated that when TAP block is added as a component in normal standard oral and parenteral pain management pain severity is further reduced up to 12 hours after a 
single short. About $50 \%$ in TAP group as compared to control group with p-value ( 2 tail) of $<0.001$. Our results are comparable with the RCT's conducted in America in 2017 concluded that TAP block was effective at different concentration in reducing post caesarean section pain even at $24 \mathrm{hrs}$ with $\mathrm{p}$-Value $0.0281[12]$.

Similar to study done in India, both pain and additional analgesic requirements were reduced with ( $\mathrm{p}$ value $<0.0001)$ at rest [13][15]. They had similar groups and used pain scores not opioids consumption to show the difference. In this study hospital post-operative pain management was not altered.

\section{Pain score on physical activities}

Efficacy of block as well is measured by improving physical function which can be obtained by assessing pain on activities such as sitting, walking, moving on bed for mother to attended the baby properly. In this study, the pain was significantly reduced in TAP group as compared to control group with statistical significance at $0,6,12,24$ hours when assessed during movement on bed including sitting, sleeping and deep breath. This was similar to study done in India and Islamabad-Pakistan which involved 60 participants, whereby TAP block showed less pain during movement resulted in earlier readiness for discharge, early ambulation, early resumption of bowel activity and was statistically significant with ( $p$ value $=0.046)[15][9]$

In Uganda RCT study comparing intrathecal morphine and TAP block showed the mean numerical rating score for intrathecal morphine vs. transversus abdominis plane blocks at $8 \mathrm{~h}, 16 \mathrm{hr}$ and $24 \mathrm{hr}$ was significantly reduced for movement and cough in transversus abdominis plane blocks different from this study at $24 \mathrm{hrs}$ [16]. In this study it was not statistically significant at all time when cough pain score was high on both groups. This could be because the pain of cesarean section essentially described into acute moderate to severe, has basically two components somatic (from abdominal wall incision) and visceral (from the uterus) which TAP act on somatic [14].

\section{Patients' satisfaction}

This study was able to show patients were satisfied with TAP block at $95.1 \%$ with statistical significance of ( $p$-value < 0.001) Similarly to study done in America the mean satisfaction levels were significantly higher in each of the bupivacaine groups about $91.5 \%$ when compared to the placebo group at $24 \mathrm{~h}$ and $48 \mathrm{~h}$. There was no difference between the two bupivacaine groups [12].

A study done in India 2015 and 2018 satisfaction score in TAP group was higher than the control group ( $p$ value $<0.001$ ) reasons being reduces opioid requirement, improves pain score, decreases sedation, promotes early ambulation as they could feed and attend their babies. (Singh et al., 2015; [15]. However, Klasen et al., 2016 showed there was patients' dissatisfaction. The main reasons could be of excessive duration of intravenous morphine administration and their effects on them which are not related to TAP.

\section{Conclusion}


This study observed the analgesic benefit of TAP block when employed with standard postoperative analgesia after cesarean section. Somatic pain was very well controlled by TAP block and visceral pain wasn't controlled by TAP this is evident by having higher scores on coughing for both groups with no statistical difference.

TAP block has the potential to become a vital component in managing postoperative pain of cesarean delivery as it is easy to perform, is safe and has definite clinical utility. Should be added in multimodal approach and not to stand alone. Despite scarcity of resource especially special needles for blocks.

From this study am recommending that TAP block should be added into pain management protocol. More comparative studies with a large sample sizes to assess the safety of the block. More comparative studies with different methodology should be done to compare different dose concentration to have a standard dose in our protocol and publish the results. Because focus now is reduction of opioids consumption, future studies are required to focus on the benefit/cost balance in these new techniques in our hospital.

\section{List Of Abbreviation}

C/S - Caesarean Section

LAST - local anaesthesia systemic toxicity

NRS - Numerical Rating Score

TAP - Trans Abdominis Plane

PACU - Post Anaesthesia Care Unity

\section{Declarations}

\section{Ethical approval and consent}

Ethical clearance with Certificate no 2394 by KCMUCo Research Ethics Review board was obtained. Informed written consent was obtained from the participants. They were informed that they can refuse to consent or withdraw consent at any point before or during the intervention without prejudicing their rights for further treatment and care based on the standard protocols at KCMC. Codes were used and not names for privacy and confidentiality on the information obtained.

\section{Consent for publication}

Not applicable

\section{Availability of data and materials}


The data set is with personal investigator it will be proved if necessarily need.

\section{Conflicts of interest}

Am declaring there is no conflicts of interest

\section{Funding}

This work was self-funded

\section{Authors' contributions}

ES conceived the study and developed the protocol, led the RCT, data analyzed and draft manuscript. RT has played a primary role in overseeing the RCT and has made a substantial contribution to the drafting of the manuscript. AN had reviewed manuscript edited it. BN contribution: Reviewed manuscript edited corrected result reporting. SS contribution: Reviewed manuscript edited and corrected. MK has made a substantial contribution to the drafting of the protocol and grammer corrections. All authors have read and approved the manuscript

\section{Acknowledgement}

We acknowledge the contribution Prof Sia Msuya and Dr. Michael Mahande academic staff of the Kilimanjaro Christian Medical University College (KCMUCo) in the department of epidemiology and biostatistics, Departments of anesthesiology and critical care and Obstetrics and Gynaecology.

\section{References}

1. I. Mylonas and K. Friese, "Indications for and Risks of Elective Cesarean Section," 2015.

2. L. Jacob, S. Taskan, G. Macharey, I. Sechet, V. Ziller, and K. Kostev, "Impact of caesarean section on mode of delivery, pregnancy-induced and pregnancy-associated disorders, and complications in the subsequent pregnancy in Germany | Auswirkungen eines kaiserschnitts auf die art der entbindung, schwangerschaftsinduzierte und," GMS Ger. Med. Sci., vol. 14, pp. 1-6, 2016.

3. N. De Carvalho Borges, L. V. Pereira, L. A. De Moura, T. C. Silva, and C. F. Pedroso, "Predictors for moderate to severe acute postoperative pain after cesarean section," Pain Res. Manag., vol. 2016, 2016.

4. F. Klasen, E. Dazeas, F. Bretelle, C. Martin, K. Baumstarck, and M. Leone, "Postoperative analgesia after caesarean section with transversus abdominis plane block or continuous infiltration wound catheter: A randomized clinical trial . TAP vs . infiltration after caesarean section," Anaesth Crit. Care Pain Med, vol. 35, pp. 411-416, 2016.

5. M. Rucklidge and E. Beattie, "Rectus sheath catheter analgesia for patients undergoing laparotomy," BJA Education, vol. 18, no. 6. Elsevier Ltd, pp. 166-172, 2018. 
6. M. M. Toro, S. John, and A. R. Faruqui, "Pattern of use of analgesics in post-operative pain management in adults undergoing laparotomy surgery: a prospective observational study," Int. Surg. J., vol. 5, no. 2, p. 662, Jan. 2018.

7. R. Karlsten, K. Ström, and L. Gunningberg, "Improving assessment of postoperative pain in surgical wards by education and training," Qual. Saf. Heal. Care, vol. 14, no. 5, pp. 332-335, 2005.

8. R. Chou et al., "Management of postoperative pain: A clinical practice guideline from the American pain society, the American society of regional anesthesia and pain medicine, and the American society of anesthesiologists' committee on regional anesthesia, executive commi," J. Pain, vol. 17, no. 2, pp. 131-157, 2016.

9. A. Kintu et al., "Postoperative pain after cesarean section: assessment and management in a tertiary hospital in a low-income country," BMC Health Serv. Res., vol. 19, no. 1, p. 68, Dec. 2019.

10. M. McGillion et al., "The Postoperative Pain Assessment Skills Pilot Trial," Pain Res. Manag., vol. 16, no. 6, pp. 433-439, 2011.

11. F. Farooq, R. Khan, and A. Ahmed, "Assessment of patient satisfaction with acute pain management service: Monitoring quality of care in clinical setting," Indian J. Anaesth., vol. 60, no. 4, pp. 248-252, 2016.

12. B. P, F. J, W. J, Y. J, and S. J, "A Randomized Controlled Trial Comparing the Efficacy of the Transversus Abdominus Plane Block with Two Concentrations of Bupivacaine in Patients Undergoing Cesarean Delivery," J. Anesth. Clin. Res., vol. 08, no. 08, pp. 6-10, 2017.

13. A. Jadon et al., "Role of ultrasound guided transversus abdominis plane block as a component of multimodal analgesic regimen for lower segment caesarean section: A randomized double blind clinical study," BMC Anesthesiol., vol. 18, no. 1, pp. 1-7, 2018.

14. T. Singh et al., "Efficacy of trans abdominis plane block for post cesarean delivery analgesia: A double-blind, randomized trial," Saudi J. Anaesth., vol. 9, no. 3, p. 298, 2015.

15. T. Mittal, A. Dey, R. Siddhartha, A. Nali, B. Sharma, and V. Malik, "Efficacy of ultrasound-guided transversus abdominis plane (TAP) block for postoperative analgesia in laparoscopic gastric sleeve resection: a randomized single blinded case control study," Surg. Endosc., vol. 32, no. 12, pp. 49854989, 2018.

16. A. Kwikiriza, J. K. Kiwanuka, P. G. Firth, M. A. Hoeft, V. E. Modest, and S. S. Ttendo, "The analgesic effects of intrathecal morphine in comparison with ultrasound-guided transversus abdominis plane block after caesarean section: a randomised controlled trial at a Ugandan regional referral hospital," Assoc. Anaesth., vol. 74, pp. 142-145, Nov. 2018.

\section{Figures}




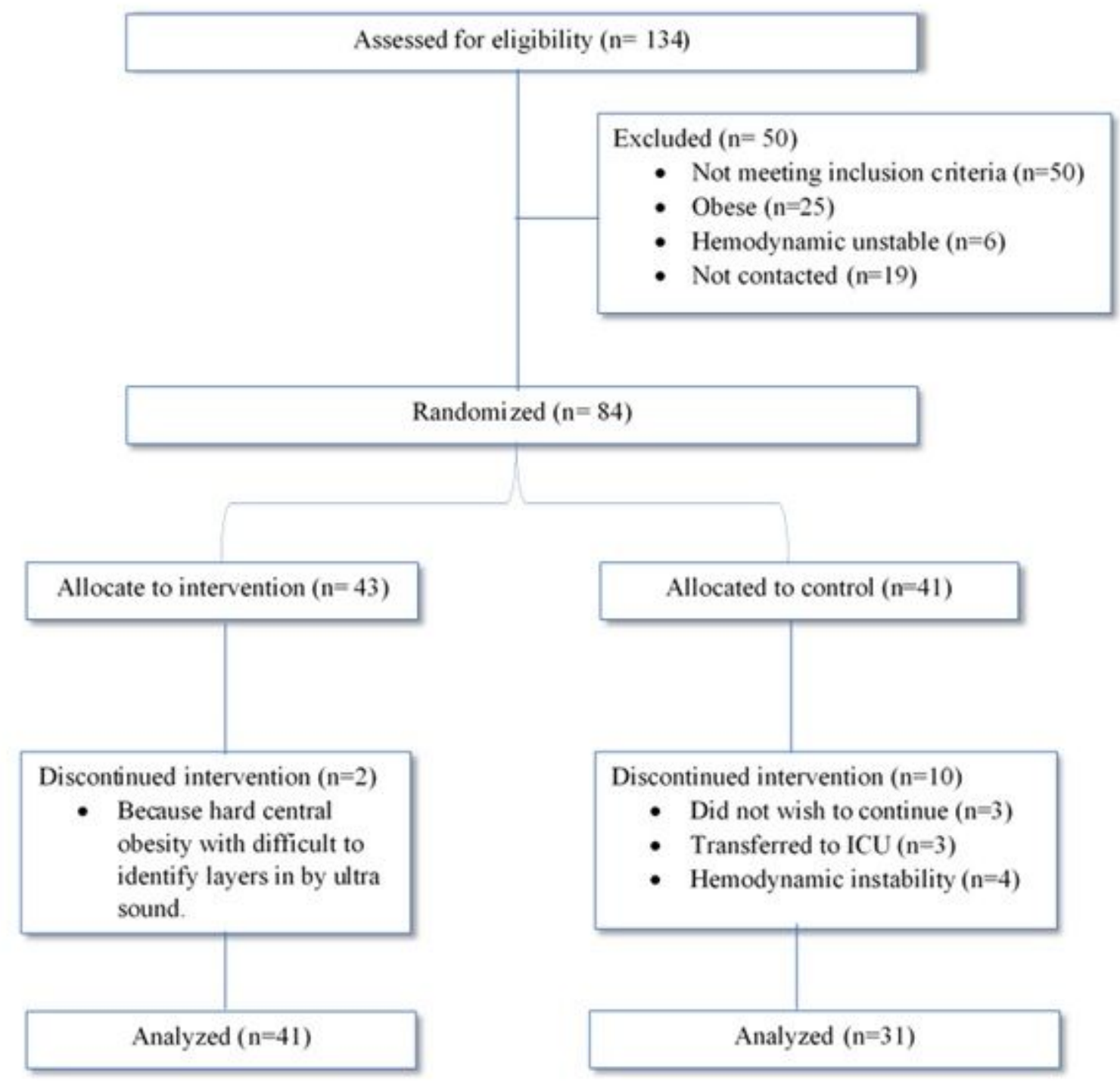

Figure 1

Flow chart 


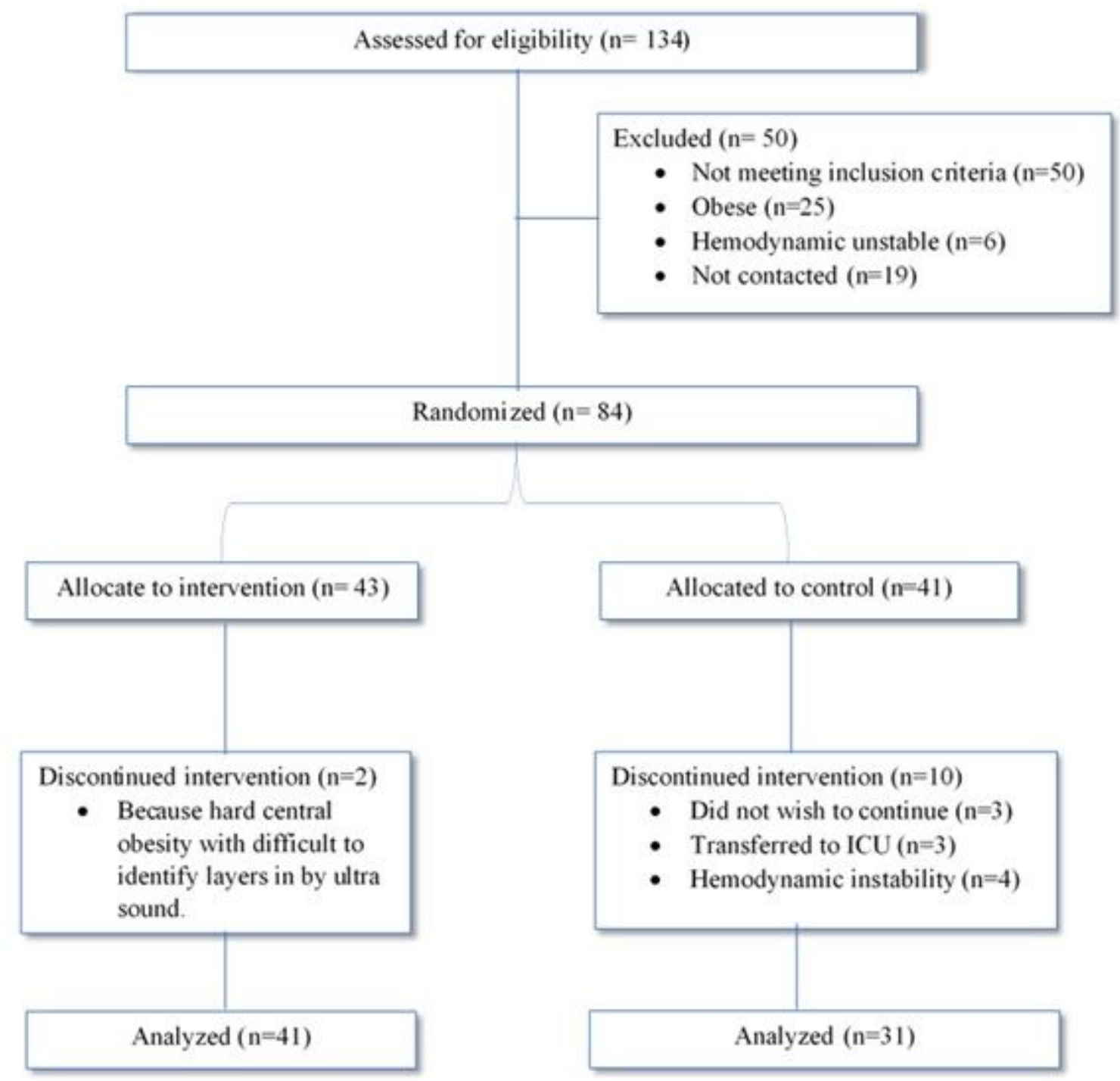

Figure 1

Flow chart 
The graph comparing level of satisafaction between the groups among participants after $24 \mathrm{hrs}$ pain management

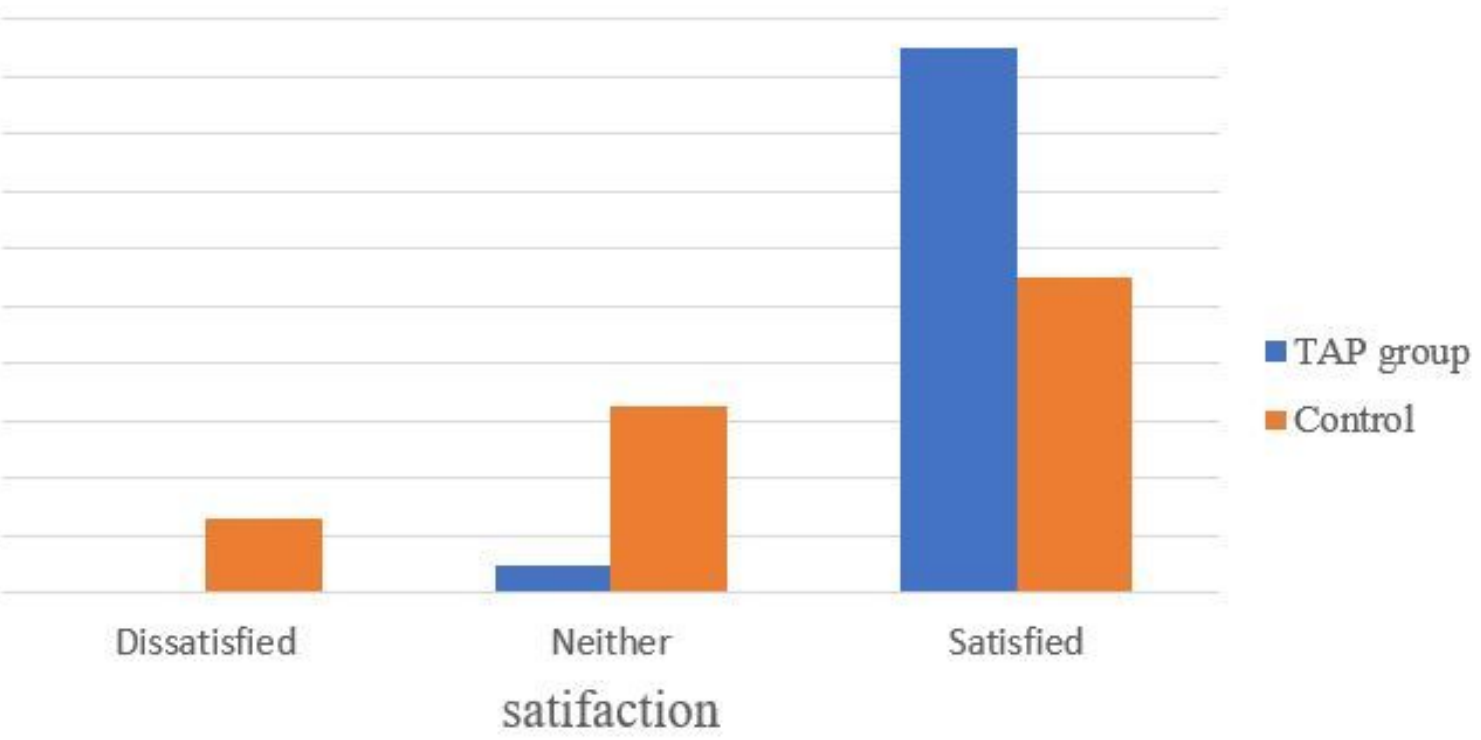

\section{Figure 2}

Comparing level of satisfaction between the groups among participants after $24 \mathrm{hrs}$ pain management

The graph comparing level of satisafaction between the

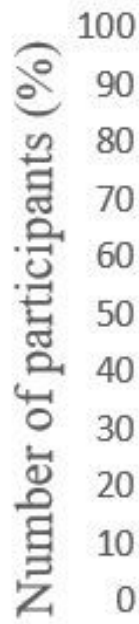
groups among participants after $24 \mathrm{hrs}$ pain management

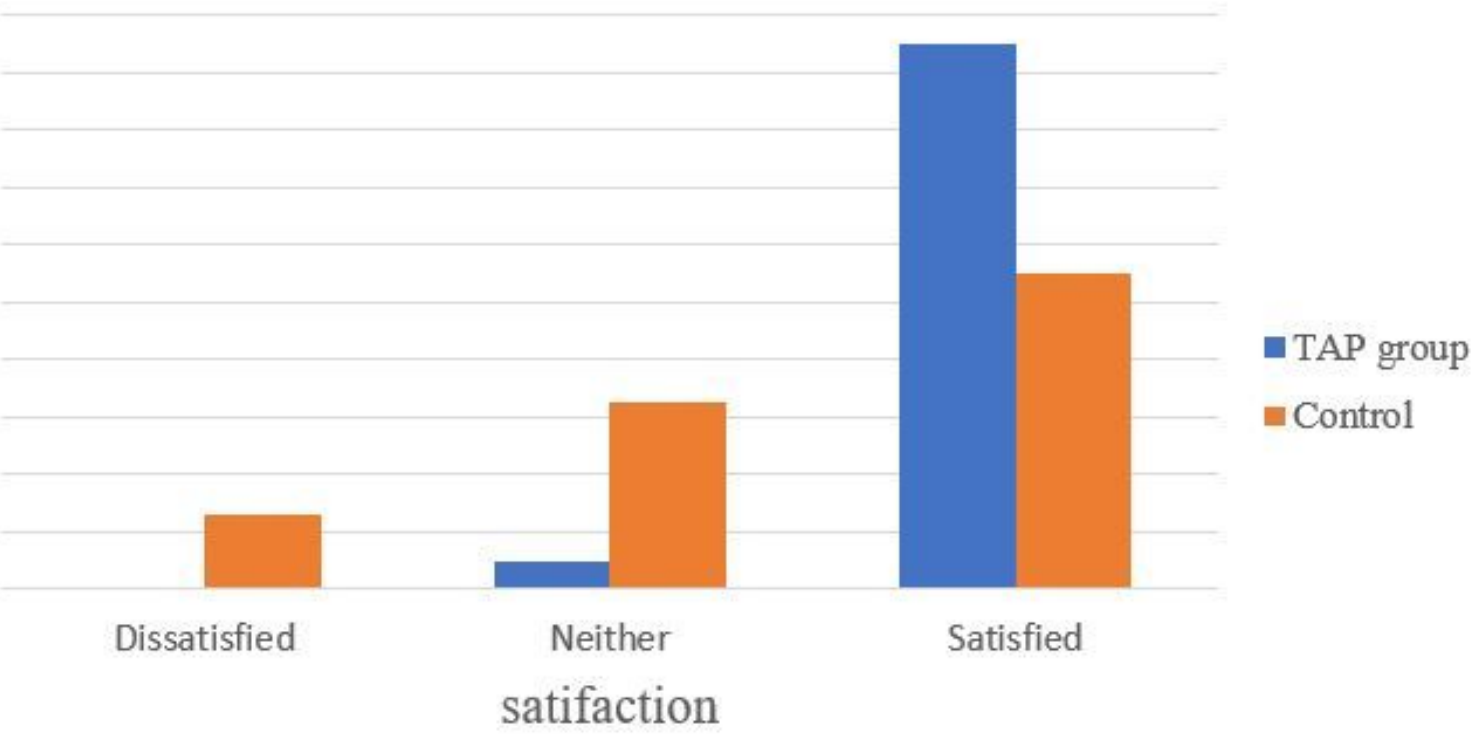

Figure 2

Comparing level of satisfaction between the groups among participants after $24 \mathrm{hrs}$ pain management 